\title{
HISTOLOGICAL STUDY OF LATE PHASE FLUORESCENCE IN THE RABBIT FUNDUS*†
}

\author{
BY
}

\author{
A. W. SOLLOM AND I. A. R. BROWN \\ Birmingham and Midland Eye Hospital
}

MANY different explanations have been put forward to account for the phenomenon of late-phase fluorescence in fluorescein fundus photography. Until the exact mechanism is known, its value as a tool for the diagnosis of fundus pathology is limited. It seems quite clear that localization of fluorescein does occur in spite of the statement by Schiller, Schayer, and Hess (1953) that aqueous fluorescein sodium diffuses everywhere so quickly after intravenous injection that it can have no localizing value. It was felt that a new approach was needed to unravel some of the apparent contradictions in the interpretation of persistent fluorescence.

We have already shown (Sollom and Brown, 1967) that a reliable experimental technique for producing tissue damage in rabbits is the application of trans-scleral diathermy. The subsequent events provide a range of pathological changes for study, in many ways similar to conditions in man, which have been investigated by the technique of fluorescein photography. In rabbits the diathermized area of fundus shows late-phase fluorescence throughout all stages of the healing process. In the experimental animal the lesion which fluoresces can be examined after enucleation to obtain a pathologist's report, whereas this is rarely possible in clinical practice. Moreover, routine processing in the pathology department can be performed in parallel with special examination of the second eye, when both have diathermy lesions at identical stages of healing. The present work is concerned with the special examination of rabbit eyes at intervals after trans-scleral diathermy.

Our earlier work has shown that the picture of fluorescence in diathermy lesions does not materially alter between 5 and 15 minutes after the intravenous injection of $0.4 \mathrm{ml}$. of a 10 per cent. solution of aqueous fluorescein. In a series of 36 rabbits, diathermy lesions were produced and all showed a similar pattern of late fluorescence. It was decided to inject the standard dose of fluorescein, wait 5 minutes, kill the animal with an overdose of intravenous barbiturate, and immediately enucleate the eyes. After identification the lesion would be prepared for frozen section. Immediate examination by fluorescence microscopy, using suitable filters, might show the characteristic green emission of fluorescein. Few guiding references could be found in the literature. Fluorescent tracing is a technique that is usually carried out in vitro and involves the use of fluorescein isothiocyanate conjugated with one of the plasma proteins. Flurorescence microscopy of the eye after intravenous fluorescein

* Received for publication April 27, 1967.

$\dagger$ Address for reprints: Birmingham and Midland Eye Hospital, Church Street, Birmingham, 3. 
does not appear to have been performed since the technique for fundus photography came into use, although the ocular tissues were examined after fluorescein injection as early as 1926 (Thiel, quoted by Ellinger, 1940). Analysis of this procedure requires caution and some of the complexities of the situation will be mentioned.

Fluorescence is a phenomenon in which the absorption of energy raises a molecule from a ground state to an excited state. The molecule then decays from the excited state with emission of light of a characteristic wavelength. The emission peak for fluorescein is $5180 \AA-\mathrm{a}$ brilliant green colour. Fluorescence depends on the molecular structure, and the characteristics vary with $\mathrm{pH}$ and temperature. The presence of various chemical groups, such as halide ions and heavy metals, may quench fluorescence; most histological stains quench fluorescence or are themselves fluorescent. The fading of fluorescence which occurs after prolonged stimulation with ultra-violet light, is thought to be a photo-chemical decomposition. The fluorescent object acts as a light source, and is best seen against a black background. In obtaining these conditions, ultra-violet light is used to excite fluorescence after passing through filters to allow transmission of selected wavelengths, primary illumination of the object being prevented by the use of a dark-ground condenser. Further barrier filters are incorporated to produce the best contrast between green fluorescein emission and autofluorescence, which occurs in most biological tissues. Autofluorescence is most commonly seen as weak blue fluorescence originating in cytoplasm, and it is intensified by most methods of preparing specimens. Connective tissue fluoresces blue/white; porphyrins red. Some cytoplasmic granules fluoresce yellow, as do some vitamins and some hormones-vitamin A has a green or yellow emission which fades rapidly under ultra-violet irradiation. Jancsø and Jancsø (1936) found that light-adapted rats showed a bright fluorescence in the retina which was not present in rats kept in the dark and they ascribed this to the presence of vitamin A. Von Euler (1935) found that fluorescence in the retinal pigment layer was caused by riboflavin, which fluoresces yellow/green and is non-fading (quoted by Nairn, 1964; Ellinger, 1940).

Trials show that fluorescein fluorescence in the structure of the eye is best seen under conditions of dark-ground illumination with passage of some contrasting light to show up pigment in the choroid. Colour transparencies are acknowledged to provide the best record and in this work high speed Ektachrome daylight film, 160 ASA, was used in conjunction with a Reichert microscope using filters $\mathrm{E}_{2}$ and $\mathrm{SP}_{2}$. The photographic image is of a high contrast but low luminosity so that long exposures are required. Most of this work was carried out at a magnification of $\times 200$ for which the exposure time was 10 minutes.

\section{Method}

Rabbits of both sexes, but not albinos, were taken after preliminary trans-scleral diathermy to both eyes as described previously (Sollom and Brown, 1967). After a variable delay of up to 9 weeks after diathermy, $0.3 \mathrm{ml}$. of 10 per cent. aqueous fluorescein was injected intravenously. This dose was found to give good late-phase fluorescence in our earlier work when satisfactory photographs were obtained 5 minutes after injection. After the same time interval the animals were killed with an overdose of intravenous pentobarbitone sodium (Nembutal), and both eyes were immediately enucleated. At each stage of healing 
one was placed in 10 per cent. formol saline for routine laboratory staining; the other used for immediate microphotography. This second eye was opened at the ora serrata and the small diathermy lesion located with a dissecting microscope. This portion of the eye was then frozen using carbon dioxide snow and, while solid, trimmed and mounted between slices of fresh liver. Frozen sections were made using a cryostat at $-25^{\circ} \mathrm{C}$. It was found necessary to make relatively thick sections, those thinner than $10 \mu$ frequently splitting when cut, so that consistent results could not be obtained. The quality of the section was then immediately checked by low-power light microscopy and suitable specimens were mounted in glycerol to be examined for fluorescence under dark-ground conditions. This whole process was carried out rapidly, the lesion being frozen within 5 minutes of enucleation and mounted for microscopy within 20 minutes. A series of colour photographs was then taken, and, after the viewing system had been altered to phase contrast, further pictures were taken of the same field.

This was found to be useful in the early stages of the work as an aid to interpretation of the picture of fluorescence, but was not as satisfactory as subsequent comparison with sections stained by normal methods.

\section{Results}

The technique of cutting frozen sections is recognized to be difficult. One of the common problems, that can only partly be avoided, is ice crystal formation which results in splitting of tissues. However, as fundus photography is concerned with details which are visible with low levels of magnification, we were mainly concerned with the appearance at levels of magnification at which the presence of some artefacts could be accepted. Injection of the rabbit with more than $0.4 \mathrm{ml}$. fluorescein produced flooding of all the tissues. The fluorescein stain in a mounted section rapidly diffuses on thawing to cover the entire field and, in fact, some of the fluorescein photographs were slightly blurred because of this after the section had been on the microscope stage for upwards of half an hour. To obviate this, it was decided that each section should be replaced with a freshly cut one after three exposures for photography-the block being kept frozen throughout.

We examined fifty eyes from thirty rabbits in this way and found that in every case fluorescein fluorescence was present only in the sclera (Fig. 1, overleaf). No other pattern of staining was seen. There was no fluorescein in the choroid or retina. Fluorescein was usually found through the whole depth of the sclera, but sometimes the fluorescence was mainly seen in the scleral layers adjacent to the choroid, suggesting that the fluorescein slowly penetrates the sclera from the choroid. Under the selected conditions of illumination, the choroidal pigment showed up red against a black background, while the retina showed a faint barely visible autofluorescence sometimes punctuated by bright star-like fluorescent granules mainly in the pigment epithelium which were probably due to vitamin A or riboflavine.

Autofluorescence was easily distinguishable from fluorescein fluorescence. Several diathermized rabbit eyes were examined without injection of the dye as controls and, in these cases, the characteristics of the faint pale blue autofluorescence were clearly established. Fluorescein-stained specimens showed the familiar shade of green coming through strongly. In some sections, when the sclera was incompletely stained by fluorescein, the contrast with autofluorescence was immediately apparent (Fig. 2, overleaf). 
Microscopical examination was carried out at regular intervals after diathermy. Comparison was made between the appearance of the lesions at identical stages of healing after fluorescein injection, and after staining with haematoxylin and eosin (ten eyes being put aside for this purpose).

The pathologist's report on the changes in the diathermy lesion one week after its production was as follows:

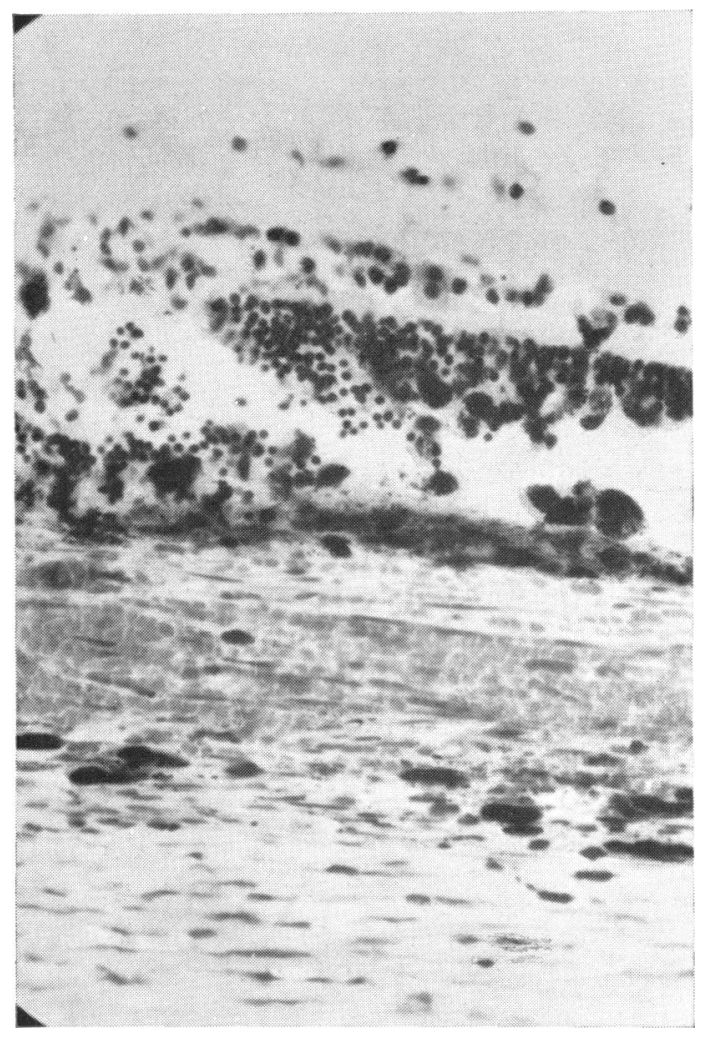

FIG. 3.-Retinal degeneration and choroidal haemorrhage 7 days after diathermy. $\times 450$.
"The retina, cytologically normal but detached beyond the periphery of the lesion, has undergone disorganization and partial destruction, especially at the peripheral limits of the lesion. In the centre the retina shows an absence of ganglion cells, but the nuclear layers are partly preserved and visual cells can still be made out in the centre, but pigment from the pigment epithelium has proliferated and is invading the retina. The choroid is haemorrhagic, thickened, and hypercellular, and choroidal pigment cells are apparently migrating into the inner scleral lamellae, where there is an excess of scleral nucleated elements." (Fig. 3)

Previous fluorescein fundus photography of a lesion at this stage showed a ring of fluorescence at the periphery of the lesion but not in the centre. Fluorescence microscopy showed fluorescein to be present only in the sclera, across the lesion and on all sides of it in a uniform manner. There was no evidence of fluorescein fluorescence from the choroid or the retina either in the lesion or in adjacent areas.

At the 2-week stage a further pathology report was obtained:

"The retina, normal outside the lesion, becomes a glial membrane, thicker in the centre of the lesion, containing proliferative pigment epithelium lining the choroid which is scarred, hypercellular, and hypovascular, and contains large round and oval pigment cells."

The sclera overlying the lesion was within normal limits (Fig. 4, overleaf). Photography of a lesion at this stage after intravenous fluorescein injection has shown a widening peripheral ring of fluorescence encroaching on the dark centre. Ultraviolet light microscopy again showed uptake of fluorescein by the sclera throughout the entire length of the section $(8-10 \mathrm{~mm}$.). There was no change in the pattern of fluorescence at any point, and the lesion could be located only by the disorganization of the choroid as shown by the contrast illumination. There was no fluorescein in the choroid or retina. 


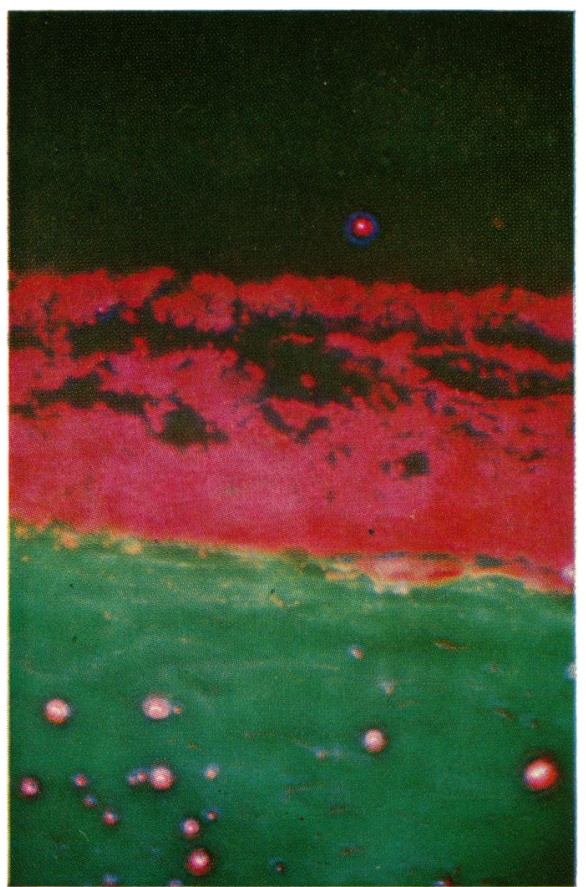

Fig. 1.-Diathermy lesion. Fluorescein staining in the sclera 7 days later. $\times 425$.

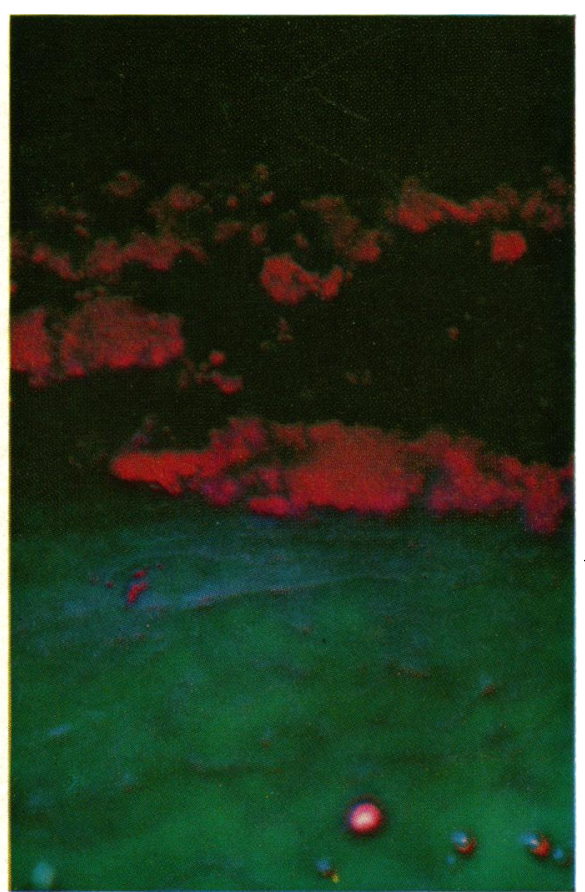

FIG. 2.-Diathermy lesion. Fluorescein staining in the sclera 35 days later. Blue autofluorescence is clearly distinguishable from fluorescein. $\times 425$. 


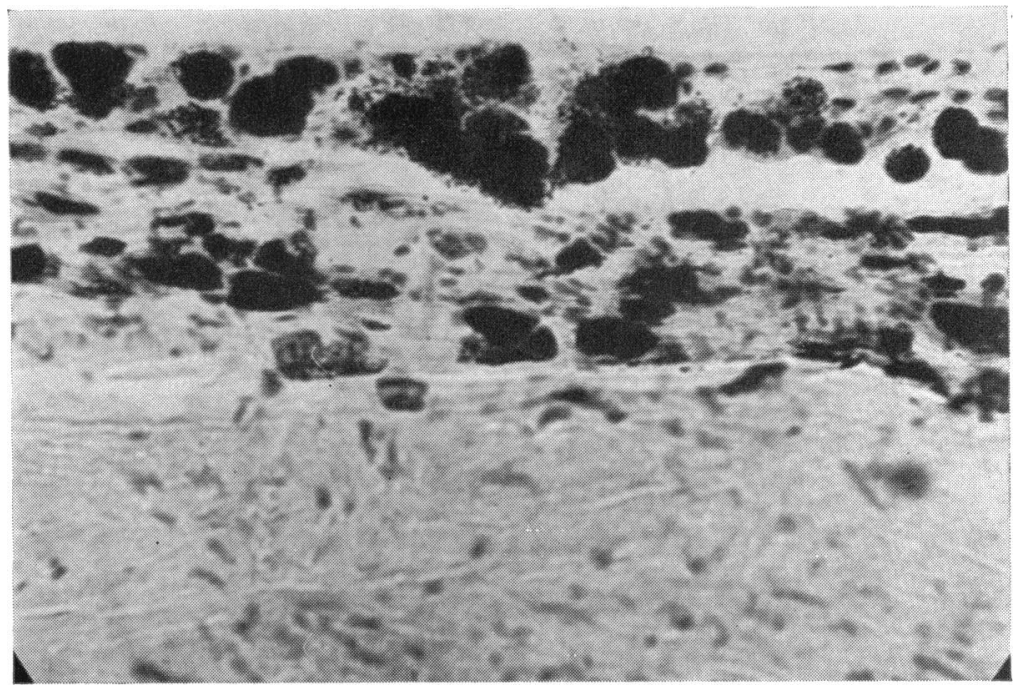

FIG. 4.-Formation of a glial membrane with choroidal hypovascularity and pigment clumping 14 days after diathermy. $\times 450$.

After 3 weeks a similar study was made and the report by the hospital pathologist stated:

"The retina is almost completely absent over the centre of the lesion while the choroid shows scarring and abnormal distribution of rounded pigment cells, but it is not thicker than normal." (Fig. 5, and Figs 6 and 7, overleaf).

Fundus photographs at this stage show late-phase fluorescence everywhere in the lesion except in a gradually diminishing central area. Microscopy after fluorescein injection shows a uniform distribution of fluorescein throughout the sclera with no

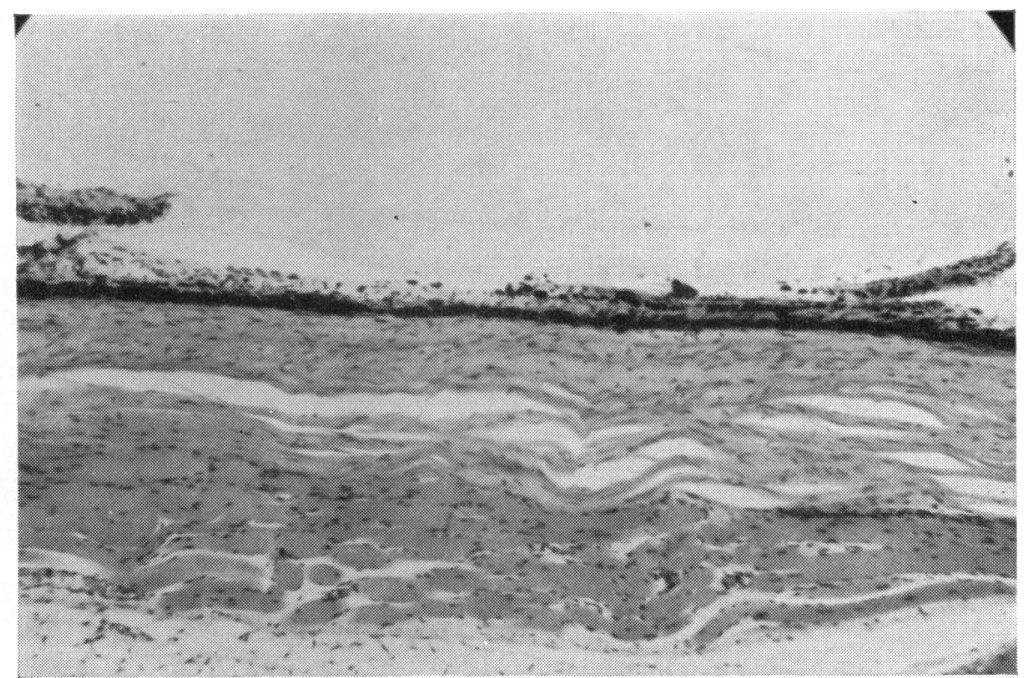

FIG. 5.- Retinal destruction and choroidal pigment changes 3 weeks after diathermy. $\times 110$. 

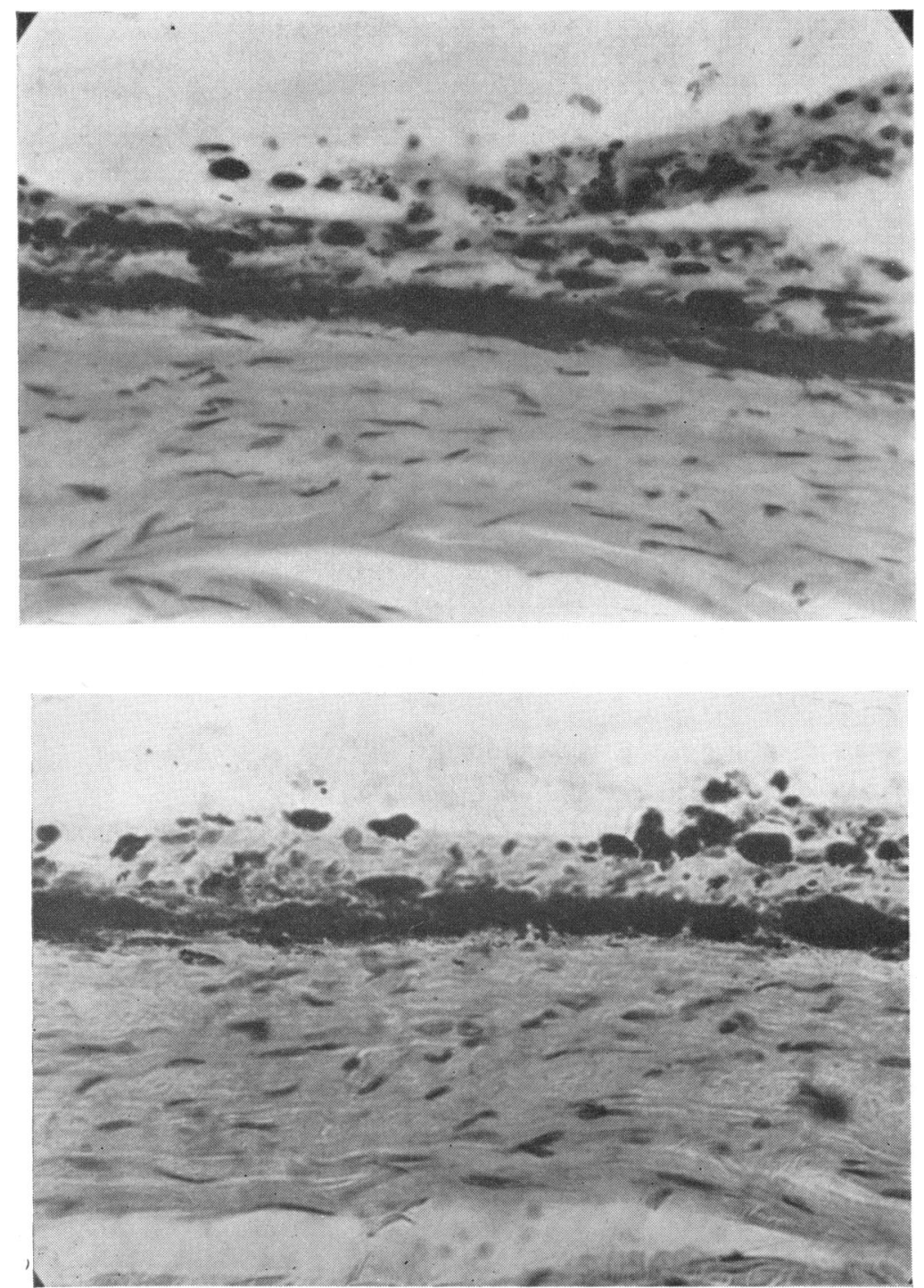

Figs 6 and 7.-High-power views of Fig. 5 to show pigment disturbance. $\quad \times 450$.

alteration at the site of the lesion which, again, can be found only by searching for changes in the choroid.

A further histological report after 5 weeks was substantially the same (Fig. 8, opposite), while the fluorescence study confirmed the previous findings. Additional studies of the fluorescence at intervals of up to 2 months after diathermy only served to support the appearances described above. In all lesions, the choroid was hypovascular, and there was disturbance of the pigment epithelium with the formation of pigment clumps.

In some sections it was felt that there was less fluorescein in the sclera at the site of the diathermy burn, while other areas showed the normal uptake. This may be due 


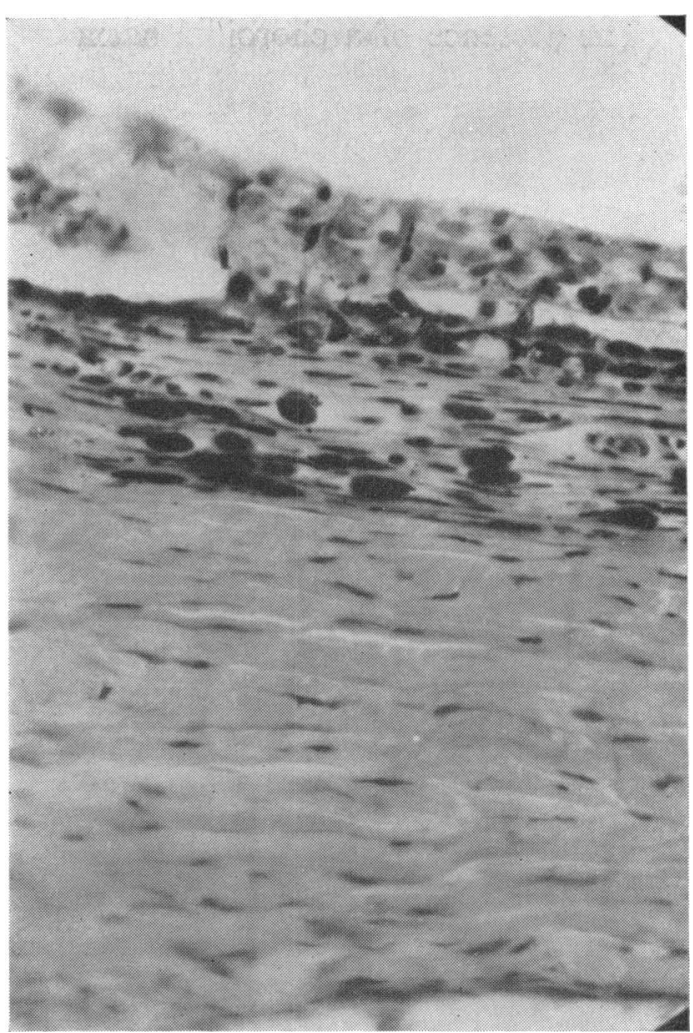

FIG. 8.-Similar changes after 36 days; compare with Fig. 2.

either to an alteration in the chemical structure or to localized destruction of the choroidal circulation cutting down the supply of fluorescein.

\section{Conclusions}

The possibility that fluorescence seen in fundus photographs may originate behind a lesion rather than in it appears to have largely been overlooked in the literature. We have repeatedly studied microscope sections of diathermy lesions in rabbit eyes, and have found that 5 minutes after injecting fluorescein the dye is apparent only in the sclera. In sections in which fluorescein staining was not uniform autofluorescence was visible. On occasion faint punctate autofluorescence presumably due to vitamin A was visible in the retina. There was no fluorescence in the choroid or Bruch's membrane. The complete absence of fluorescence from the retina suggests that there was no fluorescein in the choroid at the time of enucleation. It seems that the dye must reach the sclera from the choroidal circulation, and must be retained there possibly in a non-specific manner depending on inter-molecular electrostatic forces. Under the microscope, staining is observed in damaged and healthy sclera alike. There is no local alteration in fluorescein uptake in the lesion to correspond to the localization of late-phase fluorescence as seen in a fundus photograph. This strongly suggests that the late fluorescence shown by these lesions in fundus photography is visible because the filtering barriers of pigment and blood in the choroid 
have been deranged. The presence of a choroidal haemorrhage observed microscopically (Fig. 3) explains the lack of fluorescence in the centre of those lesions which had been observed previously.

\section{Summary}

Late-phase fluorescence of diathermy lesions in rabbit eyes 5 minutes after the injection of fluorescein has been shown to be located in the sclera. Examination of frozen sections from enucleated eyes was performed by the technique of dark-ground fluorescence microscopy.

We wish to thank the Birmingham Regional Hospital Board for the grant which made this work possible. We are also grateful to Dr. D. R. Barry, consultant pathologist, for his help and advice; to Mr. S. J. Crews for permission to use the Research Department of the Birmingham and Midland Eye Hospital; to Prof. G. Slaney, Department of Surgery, Birmingham University; and to Mr. T. F. Dee of the photographic department of the Queen Elizabeth Hospital, Birmingham.

\section{REFERENCES}

Ellinger, P. (1940). Biol. Rev., 15, 323.

NaIRN, R. C. (1964). "Fluorescent Protein Tracing", 2nd ed. Livingstone, Edinburgh.

Schiller, A. A., Schayer, R. W., and Hess, E. L. (1953). J. gen. Physiol., 36, 489.

Sollom, A. W., and Brown, I. A. R. (1967). Brit. J. Ophthal., 51, 815. 